\section{2-S1.04 EFFECTIVE ART, VIRAL LOAD AND SEXUAL BEHAVIOUR OF HIV POSITIVE MEN WHO HAVE SEX WITH MEN: CHANGES FROM 2000 TO 2010}

doi:10.1136/sextrans-2011-050109.70

${ }^{1} \mathrm{~S}$ Wayal, ${ }^{2} \mathrm{~J}$ Cassell, ${ }^{1} \mathrm{~A}$ Copas, ${ }^{1} \mathrm{C}$ Mercer, ${ }^{3} \mathrm{~S}$ Edwards, ${ }^{1} \mathrm{G}$ Hart. ${ }^{1} \mathrm{UCL}$, London, UK; ${ }^{2}$ Brighton and Sussex Medical School, Brighton, UK; ${ }^{3}$ Mortimer Market Centre, NHS Camden PCT, UK

Background Early initiation of combination antiretroviral treatment (ART) is being considered as a HIV prevention strategy. Thus, it is important to examine the association between ART, viral load (VL) and the sexual behaviour of HIV+ve patients. We compared the reporting of unprotected anal sex (UAI) in 2010 and 2000 among HIV+ve men who have sex with men (MSM) and its association with VL.

Methods HIV+ve MSM attending a central London HIV clinic were recruited in cross-sectional surveys in 2000 (411 men) and 2010 (423 men). Data on recent plasma VL (detectable (DVL) or undetectable (UVL)); ART status (treatment naïve or receiving ART); UAI with a boyfriend (main partner) and casual partners in the last 6 and 12 months respectively; boyfriend's HIV status (unknown/negative, or positive) were collected. Nonconcordant UAI (ncUAI) was defined as UAI with unknown/negative HIV status partner(s) and concordant UAI (cUAI) as UAI only with HIV+ve partner(s). Men reporting cUAI and ncUAI were treated as engaging in ncUAI. OR for 2010 compared to 2000 for UAI with casual partners were adjusted for age and DVL/UVL. Analysis for UAI with a boyfriend was adjusted also for boyfriend's HIV status and is restricted to those reporting a boyfriend.

Results Compared to 2000, 2010 respondents were more likely to be on ART with UVL (76\% vs $40 \%)$, but less likely to be on ART with DVL $(6 \%$ vs $32 \%)$ or treatment naïve (19\% vs $28 \%), p<0.001$. They were older (age $\geq 45$ years: $46 \%$ vs $22 \%, p<0.001$ ), more likely to report an HIV+ve boyfriend ( $41 \%$ vs $25 \% ; p=0.001$ ). An increase in cUAI with a boyfriend (27\% vs 14\%) remained significant after adjustment, (OR: 2.84, 95\% CI: $1.59 \%$ to $5.06 \%$; p $<0.001$ ). ncUAI with a boyfriend did not change ( $12 \%$ vs $11 \% ; \mathrm{p}=0.63)$. With regards to casual partners, cUAI decreased over time (OR: $0.50,95 \% \mathrm{CI}$ : $0.29 \%$ to $0.86 \% ; p=0.01$ ) but ncUAI increased ( $34 \%$ vs $17 \%$; OR: 2.79, $95 \%$ CI: $1.93 \%$ to $4.04 \%$; $<<0.001$ ). UVL was not associated with ncUAI with casual partners or boyfriend, but was associated with increased cUAI with casual partners (OR: 1.83, 95\% CI: $1.08 \%$ to $3.13 \% ; \mathrm{p}=0.02$ ).

Conclusions In comparison with 2000, cUAI with a boyfriend has increased, perhaps due to increased serosorting. In casual partnerships cUAI decreased and ncUAI increased. The latter has implications for onward HIV transmission and needs to be addressed by health promotion programmes. As UVL was not associated with ncUAI, these data do not suggest that early initiation of effective ART will increase HIV transmission.

\section{2-S1.05 VULNERABILITY RE-ASSESSED: THE CHANGING FACE OF SEX WORK IN GUNTUR DISTRICT, ANDHRA PRADESH}

doi:10.1136/sextrans-2011-050109.71

${ }^{1} \mathrm{~T}$ Beattie, ${ }^{2} \mathrm{~J}$ Bradley, ${ }^{3} \mathrm{U}$ Devi Vanta, ${ }^{2} \mathrm{~A}$ Shetty, ${ }^{4} \mathrm{C} \mathrm{M}$ Lowndes, ${ }^{5} \mathrm{M}$ Alary. ${ }^{1}$ London School of Hygiene and Tropical Medicine, London, UK; ${ }^{2}$ CHARME-India Project, Québec, Canada; ${ }^{3}$ Indian Institute of Health and Family Welfare IIHFW. Hyderabad, India; ${ }^{4}$ Health Protection Agency, London, UK; ${ }^{5}$ Universitaire de Québec, Quebec, Canada

Guntur district in Andhra Pradesh, south India, has over 10000 female sex workers (FSWs), of whom one fifth are estimated to be HIV infected. Following implementation of the Bill \& Melinda
Gates Foundation funded India AIDS prevention initiative, Avahan, we conducted qualitative studies with FSWs in Guntur district to examine in-depth, perceptions, behaviour and issues pertaining to their vulnerability to HIV infection. Three focus group discussions and 40 in-depth interviews were conducted with 60 FSWs, selected by random sampling. The study found evidence that client solicitation practices are changing in Guntur district, with sex workers now soliciting clients in their homes, often using mobile phones, or at their workplace, rather than in brothels or in public places. In addition, clients are most frequently requesting anal instead of vaginal sex, possibly resulting from recent exposure to pornography. FSWs were frequently unaware that unprotected anal intercourse put them at risk of HIV or other STIs, and the majority reported unprotected anal intercourse with their clients. Older FSWs and HIV-infected FSWs reported living in extreme poverty and were particularly vulnerable, often agreeing to unprotected vaginal and anal intercourse to earn enough money to survive. This, coupled with sharing of clients by home-based FSWs, suggests continued and changing vulnerability in this setting where many FSWs are already HIV infected and where understanding of risk appears deficient. Monitoring the changing sex work environment and adapting programmes accordingly will be crucial if HIV prevention programmes are to continue to reach these target populations effectively.

\section{2-S1.06 COMMUNITY RESPONSES TO AN HIV EPIDEMIC IN SOUTH INDIA: KNOWLEDGE, MORAL PANIC AND CULTURAL INERTIA}

doi:10.1136/sextrans-2011-050109.72

${ }^{1} \mathrm{~J}$ Bradley, ${ }^{2} \mathrm{~S}$ Rajaram, ${ }^{3} \mathrm{BM}$ Ramesh, ${ }^{3} \mathrm{P}$ Bhattacharjee, ${ }^{3} \mathrm{~S}$ Isac, ${ }^{3} \mathrm{~A}$ Lobo, ${ }^{4} \mathrm{~S}$ Moses, ${ }^{4} \mathrm{~J}$ Blanchard, ${ }^{3} \mathrm{R}$ Washington, ${ }^{1} \mathrm{M}$ Alary. ${ }^{1}$ Laval University, Bangalore, India; ${ }^{2} \mathrm{CHARME}$ Project, Bangalore, India; ${ }^{3}$ Karnataka Health Promotion Trust, Bangalore, India; ${ }^{4}$ University of Manitoba, Winnipeg, Canada

Background Studies have shown that as communities face serious threats to traditional values, such as that posed by HIV, cultural inertia may result, whereby existing trends towards more liberalised views of sexuality are stalled, particularly among the educated and youth. We examined changes in attitudes around HIV in Bagalkot district, south India, between 2003 and 2009

Methods General population surveys were conducted in 2003 and 2009, among approximately 6600 randomly sampled men and women in 10 villages and 20 urban blocks. Questions about HIV knowledge, sexuality, gender and condoms were included.

Results Knowledge of HIV increased from $77 \%$ in 2003 to $88 \%$ in 2009 , and condom awareness increased significantly $(37.4 \%-$ $65.4 \%$ ). However, in 2009 , only $23 \%$ of people mentioned condoms spontaneously as a means of preventing transmission (an increase from $8 \%$ in 2003). There was an increase in those who thought sex workers should be compulsorily tested for HIV $(63.0 \%$ vs $73.5 \%$, $p=0.01$ ). An increasing number of people agreed that it is wrong to talk about sex" (24.2\% vs $29.2 \%$, p=0.05), especially women $(21.9 \%$ vs $32.4 \%, p<0.01)$. There was an increase in those who thought it "wrong to talk about AIDS in a respectable family" (16.4\% vs $0.22 .1 \%, p=0.01$ ), largely among urban women, youth and the more educated, and more in 2009 thought it improper for respectable people to discuss condoms (5.5\% vs $17.9 \%, \mathrm{p}<0.01$ ). In $2003,11.8 \%$ agreed that "access to condoms promotes promiscuity", increasing to $29.5 \%$ in $2009(p<0.01)$. Men, educated people, urbanites and young people under 25 were the most likely to believe this. Sex education was thought to be equally damaging, especially among women and young people, with more agreeing that it promotes sexual activity and promiscuity in 2009 than in $2003(19.3 \%$ vs $30.2 \%, p<0.01)$. 